\title{
Propriedades tecnológicas de painéis estruturais waferboard com tratamento distilled tall oil
}

\author{
Technological properties of waferboard structural panels with distilled tall \\ oil treatment
}

\author{
Rodrigo Antunes ${ }^{1 *}$, Polliana $D^{\prime}$ Angelo Rios ${ }^{1}$, Alexsandro Bayestorff da Cunha ${ }^{1}$, \\ Rodrigo Figueiredo Terezo', Rafael Battisti Archer ${ }^{1}$, Jeimy Blanco Flórez ${ }^{1}$, \\ Helena Cristina Vieira ${ }^{1}$ e Rodrigo Buss ${ }^{1}$
}

\begin{abstract}
Resumo
O presente estudo avaliou as propriedades tecnológicas de painéis de partículas estruturais produzidos com madeira de Pinus taeda L. com diferentes tratamentos preservantes ao ataque de cupim de madeira seca Cryptotermes brevis. Foram produzidos os tratamentos: sem preservante; preservante Ciflutrina e preservante Distilled Tall Oil (DTO), utilizando adesivo fenol formaldeído e também emulsão de parafina, nas proporções de $12 \%$ e $1,5 \%$, respectivamente. Para cada tratamento foram confeccionados 5 painéis homogêneos, com densidade de $0,75 \mathrm{~g} / \mathrm{cm}^{3}$. Para a madeira, foi determinada a densidade básica e a composição química. Os painéis foram submetidos a ensaios físicos, mecânicos e de resistência ao ataque de cupim de madeira seca. A análise estatística foi realizada pelo Teste de Bartlett para homogeneidade das variâncias, Shapiro-Wilk para normalidade dos dados, teste de variância e após teste de médias de Scott-Knott ao nível de $95 \%$ de probabilidade. Os resultados demonstraram: (i) a densidade básica do Pinus taeda $\mathrm{L}$. foi de $0,356 \mathrm{~g} / \mathrm{cm}^{3}$ e a composição química foi de $2,41 \%$ de extrativos, $31,79 \%$ de lignina e $0,20 \%$ de teor de cinzas; (ii) o painel com o tratamento DTO foi o mais estável dimensionalmente para a absorção e inchamento em 2 horas, após 24 horas o mais estável foi o tratamento Ciflutrina; (iii) para as propriedades mecânicas, o tratamento DTO apresentou melhores resultados, sendo similar ao tratamento sem preservante apenas no ensaio de flexão estática (MOE); (iv) para resistência a umidade o tratamento sem preservante e o tratamento DTO apresentaram melhores resultados, tanto no ensaio cíclico quanto no ensaio em água em ebulição; $(v)$ para a resistência ao ataque de cupim de madeira seca, o tratamento Ciflutrina e o tratamento DTO obtiveram o menor desgaste em face normal. Em face bisel, o tratamento Ciflutrina obteve o menor desgaste, por ser aplicado juntamente com o adesivo. O preservante DTO obteve resultado igual ao painel sem preservante devido sua forma de aplicação, cujo método utilizado foi o pincelamento. O preservante alternativo Distilled Tall Oil apresentou-se eficiente quando comparado ao tratamento preservante comercial Ciflutrina.
\end{abstract}

Palavras chave: Preservante alternativo; Cryptotermes brevis; Pinus taeda L.

\begin{abstract}
The present study evaluated the technological properties of structural particle panels produced with Pinus taeda L. wood with different treatments preservative to the attack of dry wood termite Cryptotermes brevis. The treatments were produced: without preservative; Cyfluthrin and Preservative Distilled Tall Oil (DTO), using phenol formaldehyde adhesive and also paraffin emulsion, in the proportions of $12 \%$ and $1,5 \%$, respectively. For each treatment 5 homogeneous panels with a density of $0.75 \mathrm{~g} / \mathrm{cm}^{3}$ were made. For wood, the basic density and the chemical composition were determined. The panels were submitted to physical, mechanical and resistance tests for dry wood termite attack. The statistical analysis was performed by the Bartlett test for homogeneity of variances, Shapiro-Wilk for normality of the data, test of variance and after test of Scott-Knott averages at the 95\% probability level. The results showed that: (i) the basic density of Pinus taeda L. was $0.356 \mathrm{~g} / \mathrm{cm}^{3}$ and the chemical composition was $2.41 \%$ of extractives, $31.79 \%$ of lignin and $0.20 \%$ of ash content; (ii) the dimensional stability of the panels in 2 hours was obtained in the DTO treatment and after 24 hours in the treatment Cyfluthrin; (iii) for the mechanical properties, the DTO treatment presented better results, being similar to the treatment without preservative only in the static bending test (MOE); (iv) for wet strength the treatment without preservative and DTO treatment presented better results, both in the cyclic test and in the boiling water test; ( $v$ ) for the resistance to the attack of dry wood termite, the treatment Cyfluthrin and the treatment DTO obtained the less wear in normal face. In the bevel face,
\end{abstract}

1. Universidade do Estado de Santa Catarina - UDESC. Lages / SC, Brasil.

*Autor correspondente: eng_rodrigoantunes@hotmail.com

Sci. For., Piracicaba, v. 47, n. 123, p. 505-514, set. 2019 DOI: doi.org/10.18671/scifor.v47n123.12 
Antunes et al. - Propriedades tecnológicas de painéis estruruais waferboard

com tratamento distilled tal oil

the treatment Cyfluthrin obtained the least wear, as it was applied together with the adhesive. The DTO preservative obtained a result equal to the panel without preservative due to its form of application, whose method used was the brushing. The alternative preservative Distilled Tall Oil was efficient when compared to the commercial preservative treatment Cyfluthrin.

Keywords: Alternative preservative; Cryptotermes brevis; Pinus taeda L.

\section{INTRODUÇÃO}

Os painéis waferboard são definidos pela obtenção de partículas wafer, caracterizados por possuir propriedades iguais em todas as direções no plano do painel. Utilizados como material estrutural ou semi-estrutural, sua maior aplicação ocorre na construção civil como: assoalho, forros, revestimento de paredes, interior ou exterior. O adesivo fenol formaldeído é o mais adequado para manufatura de painéis para fins estruturais e uso exterior, aumentando a durabilidade.

Existem diversos produtos preservativos, de natureza hidrossolúvel ou oleossolúvel, para tratamento preventivo ou curativo; diversos métodos são utilizados, pressão atmosférica, pressões artificiais ou aditivos adicionados nos painéis. A combinação de produtos e processos confere ao produto padrões de qualidade diversos, adequando-a aos diferentes usos (VIDAL et al., 2015).

Diante da toxidade dos inseticidas para o homem e meio ambiente e o alto custo do controle das infestações, pesquisas são desenvolvidas em vários países com produtos e métodos alternativos para combate, como por exemplo o uso de produtos à base de materiais lignocelulósicos especialmente a lignina e os extrativos fenólicos (GALLO et. al., 2002). Pesquisas por alternativas aos sistemas tradicionais de preservação abrangem desde substâncias naturais, que são tóxicas aos organismos xilófagos, até sistemas que inibem um dos fatores: água, oxigênio ou nutrientes (BOSSARDI; BARREIROS, 2011).

Estudos como Bossardi e Barreiros (2011) e Koski (2008) focaram no desenvolvimento de alternativas aos preservantes atuais utilizando o Tall Oil. Esse atua como agente de proteção da madeira diminuindo significativamente a absorção de água capilar. Essa repelência deve-se aos seus precursores, que são extratos encontrados, principalmente, em árvores coníferas.

Os organismos que deterioram a madeira são, principalmente, fungos apodrecedores, cupins, besouros, vespas e formigas e organismos marinhos representados como alguns crustáceos e moluscos. Dentre estes organismos, os cupins de madeira seca são considerados como uma das principais pragas encontradas no Brasil. Os cupins de madeira seca Cryptotermes brevis são grandes causadores de danos em madeiras para estruturas, móveis, livros, tecidos e outros materiais lignocelulósicos em áreas urbanas de todo o mundo.

Nesse contexto, o presente trabalho tem como objetivo avaliar a utilização de diferentes tratamentos preservantes na produção de painéis estruturais com partículas do tipo "wafer" de Pinus taeda L., por meio da análise das propriedades tecnológicas e ataque de cupim de madeira seca Cryptotermes brevis.

\section{MATERIAIS E MÉTODOS}

Para realização desta pesquisa foram amostradas toras aleatórias em pilha de madeira de Pinus taeda L., plantio com idade de 8 anos, pertencentes a Fazenda Serra do Jirau, da Empresa Agroflorestal Campo Alto, em Santa Cecília/SC. Dessas pilhas, foram retiradas 6 toras com comprimento de 2,40 m, onde foram extraídos 03 discos de $30 \mathrm{~mm}$ de espessura de cada uma delas, para determinação da Densidade Básica da madeira conforme a NBR 11941 (ABNT, 2003). O restante das toras amostradas fora utilizado para geração das partículas do tipo wafer, com comprimento e espessura controlados. Parte deste material foi submetido a secagem para obtenção de umidade $3 \pm 2 \%$, com base úmida, para confecção dos painéis e parte do material foi moído em moinho tipo Willey para determinação dos componentes químicos da madeira conforme as normas relacionadas na Tabela 1.

Foram mensuradas 125 partículas aleatoriamente, partículas do tipo wafer para determinação do índice de esbeltez, razão de planicidade e área superficial, por meio da obtenção do comprimento, largura e espessura. 
Tabela 1 - Normas para determinação dos componentes químicos da madeira

Table 1 - Standards for determining the chemical components of wood

\begin{tabular}{ll}
\hline \multicolumn{1}{c}{ Análise } & \multicolumn{1}{c}{ Norma } \\
\hline Classificação do material para análise & TAPPI 264 cm-97 (TAPPI, 1997) \\
Material solúvel em hidróxido de sódio a 1\% & NBR 7990 (ABNT, 2010a) \\
Material solúvel em etanol-tolueno & NBR 14853 (ABNT, 2010b) \\
Teor de extraíveis com água & NBR 7988 (ABNT, 1984) \\
Extrativos totais & NBR 14660 (ABNT, 2004) \\
Teor de lignina & TAPPI T222 om-08 (TAPPI, 2002) \\
Teor de cinzas & TAPPI T413 om-06 (TAPPI, 1993) \\
Teor de holocelulose & Holocelulose $=100-($ extrativos totais + teor de lignina + \\
& teor de cinzas) \\
\hline
\end{tabular}

Fonte: Elaborada pelo autor, 2017.

\section{Produção dos painéis waferboard}

Foram confeccionados painéis homogêneos com densidade de $0,75 \mathrm{~g} / \mathrm{cm}^{3}$ e dimensões de $45 \mathrm{~cm}$ x $55 \mathrm{~cm}$ x $15 \mathrm{~mm}$, submetidos à prensagem com temperatura de $180^{\circ} \mathrm{C}$ e pressão específica de $40 \mathrm{kgf} / \mathrm{cm}^{2}$ pelo tempo de 08 minutos. Foram produzidos os tratamentos: testemunha (sem preservante); preservante Ciflutrina e preservante Distilled Tall Oil (DTO), utilizando adesivo fenol formaldeído e também emulsão de parafina, com base na massa seca das partículas, nas proporções de $12 \%$ e $1,5 \%$, respectivamente.

O preservante Ciflutrina foi adicionado ao adesivo e posteriormente aplicados as partículas com o uso de 46,87 $\mathrm{mL}$ de princípio ativo (Ciflutrina) por $\mathrm{m}^{3}$ de painel produzido. Para facilitar a dispersão e melhorar a uniformidade de aplicação, o princípio ativo (Ciflutrina) foi diluído a 2,50\%. O preservante DTO foi aplicado após a confecção do painel, utilizando o método de duplo pincelamento. A retenção do preservante foi monitorada após cada aplicação (pincelamento) através de pesagem. A retenção média obtida foi de 19,74 $\mathrm{kg}$ de preservante por $\mathrm{m}^{3}$ de painel.

\section{Propriedades dos painéis waferboard}

Foram avaliadas as propriedades de absorção de água e inchamento após 02 e 24 horas de imersão em água, conforme norma ASTM D 1037 (ASTM, 2002), teor de umidade, densidade aparente e taxa de não retorno em espessura, flexão estática (módulo de elasticidade e módulo de ruptura), tração perpendicular, arranque de parafuso e resistência a umidade após ensaio cíclico (avaliação do inchamento em espessura e resistência à tração perpendicular) e após o ensaio em água em ebulição (avaliação da resistência a tração perpendicular) realizados conforme norma NBR 14810-2 (ABNT, 2013).

\section{Determinação da resistência ao ataque de cupim de madeira seca}

Para realização do ensaio de resistência ao ataque de cupim de madeira seca Cryptotermes brevis, os corpos-de-prova foram encaminhados ao Instituto de Pesquisa Tecnológicas do Estado de São Paulo - IPT. Foi utilizado o Método Padrão do IPT - Publicação IPT No 1157 - Parte D/D2, 1980; Procedimento CT-FLORESTA-LAMM-EM-PE-001 "Ensaio acelerado de laboratório de resistência natural ou de madeira preservada ao ataque de térmitas do Gênero Cryptotermes (fam. Kalotermitidae)". Foram selecionados 12 corpos-de-prova com dimensões de 2,3 x 7,0 x 0,6 cm para ensaio em face normal e 12 corpos-de-prova para ensaio em fase bisel, montados dois a dois, unidos com fita adesiva e acondicionado em uma manga de vidro, presa com parafina, para delimitação da área de ataque. Para cada tratamento, foram montadas 06 réplicas, contendo 40 indivíduos de cupim, sendo 39 operários e 1 soldado. Foram utilizados também, corpos-de-prova de Pinus elliottii Engelm., como controle para avaliação da efetividade do ensaio quanto à viabilidade dos cupins utilizados. Os corpos-de-prova foram expostos aos cupins por um período de 45 dias. Ao término dos ensaios, foi avaliado o desgaste causado pelos cupins, através de notas de avaliação da intensidade do ataque, sendo: nota 0 para nenhum desgaste, nota 1 para desgaste superficial, nota 2 para desgaste moderado, nota 3 para desgaste acentuado e nota 4 para desgaste profundo (como referência os ataques aos corpos-de-prova de Pinus elliottii Engelm.). Também foi anotada a porcentagem de cupins mortos e a duração do ensaio. 
Antunes et al. - Propriedades tecnológicas de painéis estruruais waferboard

com tratamento distilled tal oil

\section{Análise estatística}

Os resultados foram submetidos a análise estatística com Teste de Bartlett para homogeneidade de variâncias, Shapiro-Wilk para normalidade dos dados, teste de variância e após teste de médias de Scott-Knott. Para o ensaio de cupim de madeira seca, os resultados do desgaste e da mortalidade foram analisados estatisticamente, em nível de significância de 5\% de probabilidade de erro, pelos seguintes métodos não paramétricos: Kruskal-Wallis para o fator amostra; e Mann-Whitney para comparação duas a duas entre amostras ensaiadas.

\section{RESULTADOS E DISCUSSÃO}

\section{Propriedades da madeira de Pinus taeda $\boldsymbol{L}$.}

O resultado médio para densidade básica da madeira de Pinus taeda L. para plantio com idade de 8 anos foi de $0,356 \mathrm{~g} / \mathrm{cm}^{3}$, semelhante aos valores obtidos pela literatura para Pinus taeda. Os valores referentes a análise química quantitativa da madeira de Pinus taeda L. estão apresentados na Tabela 2.

Os resultados médios obtidos foram semelhantes a outros pesquisadores. Mendes et al. (2002) obteve os valores de 3,03\% de extrativos totais, $28,76 \%$ de lignina e $0,22 \%$ de teor de cinzas e $67,99 \%$ para holocelulose. O mesmo autor obteve valores superiores de extrativos solúveis em água fria $(1,80 \%)$, extrativos solúveis em água quente $(2,44 \%)$ e extrativos em $\mathrm{NaOH}(8,90 \%)$.

Os teores de extrativos encontrados (Tabela 2) são baixos, portanto, possivelmente não afetam de forma significativa a polimerização do adesivo. Lima et. al. (2007) afirma que elevados teores de extrativos podem afetar as reações de polimerização do adesivo, dificultando a colagem e inviabilizando algumas aplicações.

Tabela 2 - Valores médios para composição química da madeira

Table 2 - Mean values for chemical composition of wood

\begin{tabular}{ccccccccc}
\hline \multirow{2}{*}{ Teor (\%) } & \multicolumn{1}{c}{ Extrativos } \\
\cline { 2 - 9 } & Cinzas & Totais & Etanol-Tolueno & NaOH & Água Fria & Água Quente & Lignina & Holocelulose \\
\hline \multirow{2}{*}{ Pinus taeda L. } & 0,20 & 2,41 & 0,68 & 6,43 & 0,10 & 0,42 & 31,79 & 65,60 \\
& $(0,26)$ & $(0,10)$ & $(0,21)$ & $(0,02)$ & $(0,51)$ & $(0,14)$ & $(0,03)$ & $(0,02)$ \\
\hline
\end{tabular}

Fonte: Elaborada pelo autor, 2017. Coeficiente de variação $(\mathrm{CV})$ entre parênteses.

Os teores de lignina e holocelulose demonstram que a espécie traz benefícios para produção de painéis. Conforme descrito por Iwakiri (2005) e Joseleau et al. (2004), principalmente a lignina sofre o efeito de plastificação no momento da prensagem em alta temperatura, contribuindo para adesão e melhorando as propriedades mecânicas, contribuindo também, com as propriedades de absorção de água através da redução da permeabilidade da parede celular.

O teor de cinzas encontrado reafirma os benefícios da espécie para produção de painéis, apresentando valores abaixo dos encontrados por outros autores. Iwakiri (2005) relata que o teor de cinzas na madeira encontra-se abaixo de 0,5\% e que elevados teores de cinzas podem afetar a usinabilidade devido a presença de minerais como a sílica, ocasionando desgastes excessivos de ferramentas de corte.

\section{Geometria das partículas}

Os valores obtidos de geometria das partículas estão na Tabela 3.

Dentre os valores, o IE neste estudo é próximo ao encontrado por Pereira et al. (2017) para partículas do tipo "wafer". Maloney (1993) afirmam que partículas com IE maiores normalmente resultam em aumento das propriedades de resistência a flexão estática e da estabilidade dimensional. A RP encontrada de 16,23 afeta o volume relativo de ar do colchão e a área superficial específica de 60,38 o consumo de adesivo. Valores maiores são mais desejáveis, uma vez que ocasiona uma melhora nas condições de distribuição de adesivo.

Tabela 3 - Valores médios da geometria das partículas

Table 3 - Mean values of particle geometry

\begin{tabular}{|c|c|c|c|c|c|c|c|c|c|}
\hline \multirow{2}{*}{ Espécie } & \multirow{2}{*}{$\frac{C}{(\mathrm{~mm})}$} & \multirow{2}{*}{$\frac{\mathrm{L}}{(\mathrm{mm})}$} & \multirow{2}{*}{$\frac{E}{(\mathrm{~mm})}$} & \multicolumn{2}{|c|}{ IE } & \multicolumn{2}{|c|}{ RP } & \multicolumn{2}{|c|}{ AS } \\
\hline & & & & Média & CV (\%) & Média & CV (\%) & $\left(\mathrm{cm}^{2} / \mathrm{g}\right)$ & CV (\%) \\
\hline Pinus taeda L. & 25,59 & 8,38 & 0,58 & 48,17 & 30,53 & 16,23 & 81,05 & 60,38 & 27,00 \\
\hline
\end{tabular}




\section{Propriedades físicas dos painéis waferboard}

Na Tabela 4 são apresentados os valores médios de espessura, teor de umidade, densidade e razão de compactação.

Para os resultados de espessura, o tratamento Ciflutrina que obteve o menor resultado, quando comparado aos demais tratamentos (sem preservante e DTO). Comparando os resultados obtidos por França et al. (2016), que obteve valores de espessura de 15,94 mm com espaçadores de 15,50 mm, o aumento de espessura ficou próximo do obtido no estudo.

Para os resultados de teor de umidade, observou-se diferença significativa entre todos os tratamentos, sendo o painel sem preservante o tratamento com maior teor médio de umidade (Tabela 4). Os painéis com Ciflutrina e DTO de forma geral apresentaram menor teor de umidade (11,36\% e 11,91\% respectivamente), quando comparado ao painel sem preservante (testemunha). Comparados a NBR 14801-2 (ABNT, 2013), todos os tratamentos ficaram dentro dos limites determinados quando estabilizados.

Não houve diferença significativa entre os tratamentos para os ensaios de densidade e razão de compactação. A NBR 14810-2 (ABNT, 2013) determina a tolerância em relação à densidade média de $\pm 7 \%$ em todos os tratamentos.

Os resultados de razão de compactação estão diretamente ligados a densidade dos painéis obtida no estudo. Painéis com maior razão de compactação (acima de 1,6) podem apresentar melhores propriedades de resistência mecânica, contudo sua estabilidade dimensional é prejudicada (IWAKIRI, 2005; MALONEY, 1993; MENDES et al., 2002).

Os valores médios obtidos para absorção de água, inchamento em espessura após 2 e 24 horas de imersão em água e taxa de não retorno em espessura estão apresentados na Tabela 5.

Considerando os resultados obtidos, pode-se afirmar que os tratamentos com preservante, obteve menor absorção de água tanto para imersão em 2 horas quanto para imersão em 24 horas, sendo superiores ao tratamento sem preservante.

Tabela 4 - Valores de espessura, teor de umidade, densidade e razão de compactação

Table 4 - Values of thickness, moisture content, density and compaction ratio

\begin{tabular}{|c|c|c|c|c|}
\hline \multirow{2}{*}{ Tratamento } & Espessura & TU & Densidade & RC \\
\hline & Média (mm) & Média (\%) & Média $\left(\mathrm{kg} / \mathrm{m}^{3}\right)$ & Média $\left(\mathrm{kg} / \mathrm{m}^{3}\right)$ \\
\hline Sem preservante & $15,71 \mathrm{~b}(0,80)$ & 12,43 a $(2,61)$ & 795,55 a $(2,45)$ & 2,23 a $(2,45)$ \\
\hline Ciflutrina & 15,56 a $(0,54)$ & 11,36 c $(1,66)$ & 797,40 a $(2,77)$ & 2,24 a $(2,77)$ \\
\hline Distilled Tall Oil & $15,77 \mathrm{~b}(0,42)$ & 11,91 b $(0,68)$ & 816,27 a $(2,40)$ & 2,29 a $(2,40)$ \\
\hline
\end{tabular}

NOTA: Coeficiente de variação entre parênteses. As médias seguidas de letras iguais na mesma coluna não apresentam diferença significativa entre si pelo Teste de Scott-Knott em nível de 95\% de probabilidade. Fonte: Elaborada pelo autor, 2017.

Tabela 5 - Valores de absorção de água, inchamento em espessura em 2 e 24 horas e taxa de não retorno em espessura (TNRE)

Table 5 - Water absorption values, swelling in thickness at 2 and 24 hours and non-return rate in thickness (TNRE)

\begin{tabular}{|c|c|c|c|c|c|}
\hline \multirow{3}{*}{ Tratamento } & \multicolumn{2}{|c|}{ Absorção de água } & \multicolumn{2}{|c|}{ Inchamento em espessura } & \multirow{2}{*}{ TNRE } \\
\hline & 2 horas & 24 horas & 2 horas & 24 horas & \\
\hline & Média (\%) & Média (\%) & Média (\%) & Média (\%) & Média (\%) \\
\hline Sem preservante & 11,94 a $(18,33)$ & 27,08 a $(14,57)$ & 4,14 a $(22,66)$ & 11,79 a $(9,68)$ & 10,60 a $(8,99)$ \\
\hline Ciflutrina & $7,01 \mathrm{~b}(18,99)$ & $16,44 \mathrm{c}(8,95)$ & $3,16 \mathrm{~b}(16,45)$ & $6,74 \mathrm{~b}(14,53)$ & $7,22 \mathrm{c}(11,63)$ \\
\hline Distilled Tall Oil & 5,13 c $(11,45)$ & 19,41 b $(11,65)$ & 1,92 c $(19,81)$ & 10,71 a $(3,20)$ & 8,58 b $(4,68)$ \\
\hline \multicolumn{6}{|l|}{ Referência } \\
\hline CSA 0437 (1993) & Máx. 10,00 & Máx. 15,00 & - & - & - \\
\hline USDA $(1987)^{\star}$ & - & 5,00 a 50,00 & - & - & - \\
\hline NBR 14810-2 (2013) & - & - & - & $105^{\star *}$ & - \\
\hline
\end{tabular}

NOTA: *Forest Products Laboratory. *Requisito para painéis estruturais para uso em condições severas de carga, em ambientes úmidos (Tipo P7). Coeficiente de variação entre parênteses. As médias seguidas de letras iguais na mesma coluna não apresentam diferença significativa entre si pelo Teste de Scott-Knott ao nível de 95\% de probabilidade. Fonte: Elaborada pelo autor, 2017.

Koski (2008) afirma que o grau de secagem do Tall Oil tem um efeito significativo sobre a repelência da água. O óleo após secagem fecha os capilares completamente do que o óleo natural e também evita a água capilar a longo prazo. A hidrofobia ou enchimento dos capilares retardam a taxa de absorção de água, mas a extensão da captação ainda pode ser igual à da madeira não tratada 
durante um longo período de tempo em virtude da difusão (menor prazo desempenho). Este é um fato comum que se aplica a muitos outros repelentes de água.

Para o inchamento em 2 horas de imersão, todos os tratamentos apresentaram diferença significativa, sendo o tratamento DTO o que obteve o menor inchamento $(1,92 \%)$ e o tratamento sem preservante o maior $(4,14 \%)$.

Para o inchamento em 24 horas de imersão, houve diferença significativa somente para o tratamento Ciflutrina que obteve o menor resultado de inchamento $(6,74 \%)$. Os dois outros tratamentos (sem preservante e DTO) não tiveram diferença significativa, obtendo os valores de $11,79 \%$ e 10,71\% respectivamente. Tanto o tratamento Ciflutrina e o tratamento DTO atenderam a especificação da NBR 14810-2 (ABNT, 2013).

$\mathrm{O}$ valor de inchamento significativamente menor que os demais tratamentos em 2 horas de imersão no tratamento DTO, pode ser explicado pela aplicação do preservante, que contém ácidos graxos e ácidos resínicos, atuando como repelente a água nas primeiras 2 horas do ensaio, perdendo sua eficiência após 24 horas de exposição, igualando-se ao tratamento sem preservante.

Analisando os valores de TNRE, a qual expressa o inchamento definitivo do painel, verificou-se diferença significativa entre os tratamentos, sendo o tratamento sem preservante o que se destacou de forma negativa com 10,60\%. O tratamento Ciflutrina foi melhor, obtendo um baixo valor de $7,22 \%$, consequentemente uma melhor estabilidade dimensional dos painéis

\section{Propriedades mecânicas dos painéis waferboard}

Os valores médios obtidos para flexão estática e módulo de elasticidade, tração perpendicular e arrancamento de parafuso estão apresentados na Tabela 6 .

Tabela 6 - Valores de flexão estática e módulo de elasticidade, tração perpendicular e arrancamento de parafuso

Table 6 - Static bending and modulus of elasticity, perpendicular traction and screw pulling values

\begin{tabular}{|c|c|c|c|c|c|}
\hline \multirow{3}{*}{ Tratamento } & \multicolumn{2}{|c|}{ Flexão Estática } & \multirow{2}{*}{$\begin{array}{c}\text { Tração } \\
\text { Perpendicular }\end{array}$} & \multicolumn{2}{|c|}{ Arrancamento de Parafuso } \\
\hline & MOR & MOE & & Topo & Superfície \\
\hline & Média $\left(\mathrm{N} / \mathrm{mm}^{2}\right)$ & Média $\left(\mathrm{N} / \mathrm{mm}^{2}\right)$ & Média $\left(\mathrm{N} / \mathrm{mm}^{2}\right)$ & Média (N) & Média (N) \\
\hline Sem pres & 23,68 a $(6,37)$ & $2.533,76$ b $(6,67)$ & 0,68 a $(2,18)$ & $1.728,85$ a $(8,01)$ & $1.967,95 \mathrm{~b}(3,26)$ \\
\hline Ciflutrina & 24,96 a $(11,32)$ & $2.870,87$ a $(5,44)$ & $0,65 \mathrm{~b}(3,20)$ & $1.425,20 \mathrm{~b}(17,11)$ & $1.859,95 \mathrm{~b}(11,30)$ \\
\hline Distilled Tall Oil & 25,26 a $(8,69)$ & $2.633,35$ b $(7,27)$ & 0,68 a $(2,63)$ & $1.617,25$ a $(9,75)$ & $2.160,65$ a $(10,61)$ \\
\hline
\end{tabular}

Referência

NBR 14810-2 (2013)* $\quad 20 \quad 3.100 \quad 0,70$

CS 236-66 (CS, 1968)** $\quad 17,16 \quad 3.089 \quad 0,41$

$0,41 \quad 890$

1.112

NOTA: *Requisito para painéis estruturais para uso em condições severas de carga, em ambientes úmidos (Tipo P7). **Painéis de partículas feita com adesivo durável e altamente resistente a umidade e calor (geralmente adesivo fenol formaldeído) adequada para aplicações interiores e certas aplicações exteriores - Classe 2. Coeficiente de variação entre parênteses. MOR - É o módulo de resistência à flexão estática, expresso em newtons por milímetro quadrado $\left(\mathrm{N} / \mathrm{mm}^{2}\right)$. MOE - É o módulo de elasticidade, expresso em newtons por milímetro quadrado $\left(\mathrm{N} / \mathrm{mm}^{2}\right)$. As médias seguidas de letras iguais na mesma coluna não apresentam diferença significativa entre si pelo Teste de Scott-Knott ao nível de $95 \%$ de probabilidade. Fonte: Elaborada pelo autor, 2017.

Os resultados obtidos para flexão estática (MOR) não apresentaram diferença significativa entre os tratamentos. Comparando com o requisito da NBR 14801-2 (ABNT, 2013), todos os tratamentos atenderam a especificação.

Para o módulo de elasticidade (MOE), houve diferença significativa entre o tratamento Ciflutrina e os demais (sem preservante e DTO), os painéis com Ciflutrina apresentaram maior valor $\left(2.870,97 \mathrm{~N} / \mathrm{mm}^{2}\right)$ que pode ser explicado pelo baixo teor de umidade quando comparado aos outros tratamentos. De acordo com Mendes, et al.(2013) um maior teor de umidade nos painéis pode reduzir seu módulo de elasticidade. Comparando com a NBR 14810-2 (ABNT, 2013), todos os tratamentos não atingiram o valor solicitado pela norma para caracterização do painel como Tipo P7. Avaliando as demais classificações da norma, os valores obtidos em todos os tratamentos enquadram o painel como Tipo P5 - painéis estruturais para uso em condições úmidas, sendo o valor requisitado mínimo de $2.400 \mathrm{~N} / \mathrm{mm}^{2}$.

Para os resultados de tração perpendicular, houve diferença significativa entre o tratamento Ciflutrina e os demais (sem preservante e DTO). O tratamento Ciflutrina obteve o menor valor $\left(0,65 \mathrm{~N} / \mathrm{mm}^{2}\right)$. Quando comparamos os resultados médios com a NBR 14810-2 (ABNT, 2013), para classificação 
Tipo 07, verificou-se que nenhum dos tratamentos alcançaram o valor especificado. Os tratamentos sem preservante e DTO foram os que mais se aproximaram $\left(0,68 \mathrm{~N} / \mathrm{mm}^{2}\right)$. Avaliando as outras classificações da norma, os valores obtidos em todos os tratamentos enquadram o painel como Tipo P6 - painéis estruturais para uso em condições severas de carga, em ambientes secos, sendo o valor mínimo de $0,50 \mathrm{~N} / \mathrm{mm}^{2}$.

A aplicação de 1,5\% de emulsão de parafina pode ter sido o principal fator que causou uma redução da resistência a tração perpendicular. A utilização de emulsão de parafina melhora a resistência a água nos painéis de partículas, mas é duvidosa essa melhoria também na tração perpendicular, visto que não é suficientemente forte a atração química entre a parafina e a partícula de madeira, para melhorar a adesão entre as partículas.

Para a resistência ao arrancamento de parafuso no topo (RAP-T), verificou-se diferença significativa entre o tratamento Ciflutrina, que obteve o menor valor $(1.425,20 \mathrm{~N}$ ) e os demais tratamentos (sem preservante e DTO), que obteram valores de $1.728,85 \mathrm{~N}$ e $1.617,25 \mathrm{~N}$ respectivamente.

Para a resistência ao arrancamento de parafuso na superfície (RAP-S), houve diferença significativa entre o tratamento DTO que obteve o maior valor $(2.160,65 \mathrm{~N})$ e os demais tratamentos que obteram valores menores.

Comparando com a norma CS 236-66 (CS,1968), todos os tratamentos superaram o valor mínimo para resistência ao arrancamento de parafuso no topo e na superfície, demostrando eficiência no processo produtivo dos painéis e que o DTO não altera negativamente a resistência dos painéis para essas propriedades.

\section{Resistência a umidade}

Os valores obtidos para resistência a umidade ensaio cíclico (Opção 1) e ensaio em água em ebulição (Opção 2) estão apresentados na Tabela 7.

Tabela 7 - Valores médios para inchamento e tração após ensaio cíclico e tração após ensaio em água em ebulição

Table 7 - Mean values for swelling and traction after cyclic test and traction after test in boiling water

\begin{tabular}{|c|c|c|c|}
\hline \multirow{3}{*}{ Tratamento } & \multicolumn{2}{|c|}{ Ensaio Cíclico } & \multirow{2}{*}{$\begin{array}{l}\text { Água em Ebulição } \\
\text { Tração perpendicular }\end{array}$} \\
\hline & Inchamento & Tração perpendicular & \\
\hline & Média (\%) & Média $\left(\mathrm{N} / \mathrm{mm}^{2}\right)$ & Média $\left(\mathrm{N} / \mathrm{mm}^{2}\right)$ \\
\hline Sem preservante & 15,79 a $(21,40)$ & 0,58 a $(17,57)$ & 0,59 a $(15,97)$ \\
\hline Ciflutrina & 16,00 a $(31,72)$ & $0,32 \mathrm{~b}(54,66)$ & 0,45 a $(45,50)$ \\
\hline Distilled Tall Oil & 16,12 a $(8,88)$ & 0,52 a $(13,76)$ & 0,55 a $(7,77)$ \\
\hline \multicolumn{4}{|l|}{ Referência } \\
\hline NBR 14810-2 (ABNT, 2013) & $11 \%{ }^{*}$ & $0,36^{*}$ & $0,23^{*}$ \\
\hline
\end{tabular}

NOTA: *Requisito para painéis estruturais para uso em condições severas de carga, em ambientes úmidos (Tipo P7). CV - Coeficiente de variação. As médias seguidas de letras iguais na mesma coluna não apresentam diferença significativa entre si pelo Teste de Scott-Knott ao nível de $95 \%$ de probabilidade. Fonte: Elaborada pelo autor, 2017.

Os valores obtidos após ensaio cíclico para inchamento em espessura não diferiram estatisticamente entre si. Todos os valores ficaram acima do recomendado na NBR 14810-2 (ABNT, 2013). Este resultado pode estar diretamente relacionado a razão de compactação (Tabela 4), conforme descrito por Iwakiri (2005) e Maloney (1993) que afirmam que valores acima de 1.6 podem melhorar as propriedades de resistência, mas por outro lado, o inchamento em espessura será maior devido a maior taxa de compressão exercida no material durante a prensagem do painel.

Para os valores médios obtidos para tração perpendicular após o ensaio cíclico, houve diferença significativa entre o tratamento Ciflutrina, que obteve o menor valor $\left(0,32 \mathrm{~N} / \mathrm{mm}^{2}\right)$ e os demais tratamentos (sem preservante e DTO) que obtiveram valores de 0,58 N/mm² e 0,52 N/mm² respectivamente. Avaliando a especificação da NBR 14810-2 (ABNT, 2013), apenas o tratamento com Ciflutrina não atendeu os requisitos mínimos propostos $\left(0,36 \mathrm{~N} / \mathrm{mm}^{2}\right)$.

Os valores obtidos de resistência a tração perpendicular após ensaio em água em ebulição não diferiram estatisticamente entre si. Todos os valores ficaram acima do recomendado na NBR 14810-2 (ABNT, 2013). 
Antunes et al. - Propriedades tecnológicas de painéis estruruais waferboard

com tratamento distilled tal oil

\section{Resistência ao ataque de cupim de madeira seca}

Os resultados médios do ensaio de cupim de madeira seca estão apresentados na Tabela 8 - face normal e na Tabela 9 - face bisel. Foram avaliados o desgaste, mortalidade e duração do experimento.

Avaliando o desgaste ocorrido pelo ataque dos cupins de madeira seca em face normal, o tratamento Ciflutrina foi igual ao tratamento DTO, diferindo estatisticamente da amostra controle e dos painéis sem preservante, indicando eficiência do produto aplicado no controle do ataque dos cupins.

Tabela 8 - Resultado do ensaio de cupim de madeira seca - face normal

Table 8 - Results of dry wood termite test normal face

\begin{tabular}{llccc}
\hline \multicolumn{1}{c}{ Amostra } & \multicolumn{1}{c}{ Código LAMM } & $\begin{array}{c}\text { Desgaste } \\
(*)\end{array}$ & $\begin{array}{c}\text { Mortalidade (**) } \\
(\%)\end{array}$ & $\begin{array}{c}\text { Duração } \\
\text { (dias) }\end{array}$ \\
\hline Pinus elliottii & $1755 / 17-$ CONTROLE & $4,0 \mathrm{a}$ & $35,4 \mathrm{a}$ & 45 \\
Sem preservante & $1755 / 17-01 \mathrm{~A}$ & $3,5 \mathrm{a}$ & $29,6 \mathrm{a}$ & 45 \\
Ciflutrina & $1755 / 17-02 \mathrm{~A}$ & $0,8 \mathrm{~b}$ & $60,0 \mathrm{~b}$ & 45 \\
Distilled Tall Oil & $1755 / 17-03 \mathrm{~A}$ & $0,8 \mathrm{~b}$ & $29,2 \mathrm{a}$ & 45 \\
\hline
\end{tabular}

Fonte: Relatório de Ensaio № I 094 I53 - 203, elaborado pelo LAMM/CT-FLORESTAL/IPT, 20I7. (*) - Kruskal-Wallis $\left(\mathrm{p}=1,627 \times 10^{-6}\right)$; Mann-Whitney - as médias seguidas da mesma letra na coluna não diferem estatisticamente. $\left.{ }^{*} *\right)$ - Kruskal-Wallis $(\mathrm{p}=0,0001657)$; Mann-Whitney - as médias seguidas da mesma letra na coluna não diferem estatisticamente.

Tabela 9 - Resultado do ensaio de cupim de madeira seca - face bisel

Table 9 - Results of the dry wood termite test - bevel face

\begin{tabular}{llccc}
\hline \multicolumn{1}{c}{ Amostra } & \multicolumn{1}{c}{ Código LAMM } & $\begin{array}{c}\text { Desgaste } \\
\left(^{*}\right)\end{array}$ & $\begin{array}{c}\text { Mortalidade } \\
(* *)(\%)\end{array}$ & $\begin{array}{c}\text { Duração } \\
\text { (dias) }\end{array}$ \\
\hline Pinus elliottii & $1755 / 17-$ CONTROLE & $4,0 \mathrm{a}$ & $35,4 \mathrm{a}$ & 45 \\
Sem preservante & $1755 / 17-01 \mathrm{~B}$ & $2,7 \mathrm{~b}$ & $40,0 \mathrm{a}$ & 45 \\
Ciflutrina & $1755 / 17-02 \mathrm{~B}$ & $0,0 \mathrm{c}$ & $93,8 \mathrm{~b}$ & 42 \\
Distilled Tall Oil & $1755 / 17-03 \mathrm{~B}$ & $2,7 \mathrm{~b}$ & $37,5 \mathrm{a}$ & 45 \\
\hline
\end{tabular}

Fonte: Relatório de Ensaio № I 094 I53 - 203, elaborado pelo LAMM/CT-FLORESTAL/IPT, 20I7. (*) - Kruskal-Wallis ( $\mathrm{p}=$ I,627x I0-6); Mann-Whitney - as médias seguidas da mesma letra não diferem estatisticamente. $\left(^{* *}\right)$ - Kruskal-Wallis $(\mathrm{p}=0,0001657)$; Mann-Whitney - as médias seguidas da mesma letra não diferem estatisticamente

O valor de desgaste para os tratamentos Ciflutrina e DTO foram iguais $(0,8)$, classificado entre nenhum desgaste e desgaste superficial. Já para o tratamento sem preservante e a amostra controle, que apresentaram valores de 3,5 e 4,0 respectivamente, são classificados entre desgaste acentuado e desgaste profundo.

A amostra sem preservante demonstrou a susceptibilidade ao ataque de cupim de madeira seca, confirmando a necessidade de utilização de aditivos que inibam o ataque desses agentes. $\mathrm{O}$ tratamento Ciflutrina obteve esperado desempenho e eficiência, tanto para o requisito desgaste quanto para a mortalidade dos cupins, por ser um inseticida comercialmente utilizado. O tratamento DTO obteve igual desempenho e eficiência quanto ao controle de ataque de cupim.

Sabe-se que a resistência a biodegradação é atribuída a presença de substâncias do lenho, como taninos e outras substâncias fenólicas complexas tóxicas a fungos e a insetos xilófagos, sendo os constituintes do Tall Oil.

Os resultados obtidos relacionados a estabilidade dimensional e a eficiência ao controle de ataque de cupins de madeira seca nos painéis que utilizaram o DTO como agente de proteção confirmam a afirmação de Hyvönen et al. (2006) apud Bossardi e Barreiros (2011), que o Tall Oil tem sido considerado promissor por reduzir significativamente a absorção de água, removendo um dos fatores que favorecem a madeira a ser atacada por fungos e insetos: água, oxigênio e nutrientes.

Para avaliação da mortalidade dos cupins (Tabela 8), observou-se que a amostra controle, painel sem preservante e painel com DTO foram estatisticamente iguais. Isso ocorre devido as amostras citadas, não possuírem ação inseticida, ao contrário do tratamento Ciflutrina que diferiu dos demais., por ser um piretróide, a Ciflutrina é altamente tóxica agindo no organismo dos insetos xilófagos. A baixa mortalidade dos cupins no tratamento DTO é justificada pela não toxidade do produto, composto por ácidos graxos, ácidos resínicos e insaponificáveis, extraídos da madeira, que não atuam como inseticida, mas sim como conservante natural. (BOSSARDI; BARREIROS, 2011; KOSKI, 2008).

Avaliando o desgaste ocorrido pelo ataque dos cupins de madeira seca em face bisel, foi verificado estatisticamente que o painel com Ciflutrina apresentou o menor desgaste entre os tratamentos. 
O tratamento DTO e o tratamento sem preservante foram iguais. O tratamento Ciflutrina diferiu da amostra controle e da amostra sem preservante, indicando que o aditivo está presente no interior do painel. Isso ocorre através da adição do aditivo durante o processo produtivo, com a mistura direta no adesivo, apresentando maior eficiência quando comparado ao método de pincelamento que ocorre somente na superfície do produto aplicado.

O tratamento DTO e o tratamento sem preservante apresentaram diferença da amostra controle. Este resultado pode ser atribuído a utilização de adesivo fenol formaldeído, conforme afirma Silva et. al. (2010), que comparou a influência de adesivos tânicos com adesivo fenol formaldeído sobre a resistência de painéis aglomerados de Pinus caribea ao cupim Coptotermes gestroi e concluíram que o adesivo fenol formaldeído se apresentou mais resistente.

Avaliando a taxa de mortalidade dos cupins, observou-se que o tratamento sem preservante, tratamento DTO e a amostra controle não deferiram entre si, indicando uma baixa mortalidade quando comparado ao tratamento com Ciflutrina, onde a duração média do experimento foi de 42 dias, pois ocorreu a mortalidade de 100\% dos cupins em duas das seis amostras analisadas.

\section{CONCLUSÃO}

A partir da avaliação dos resultados obtidos pode-se concluir:

A densidade básica da madeira de Pinus taeda L. foi de $0,356 \mathrm{~g} / \mathrm{cm}^{3}$ e sua composição química é benéfica para produção de painéis de partículas por possuir baixos teores de extrativos, alto teor de lignina e baixo teor de cinzas;

Para as propriedades físicas, o melhor resultado para estabilidade dimensional dos painéis (absorção e inchamento) foi o tratamento DTO após 2 horas e para o tratamento Ciflutrina, após 24 horas. Ambos os ensaios e tratamentos atenderam as normas, exceto a avaliação para absorção de água em 24 horas.

Para as propriedades mecânicas, foi evidenciado que o tratamento DTO apresentou melhores resultados nos ensaios de tração perpendicular, arranque de parafuso (topo/superfície) e MOR. Somente para o MOE o tratamento com Ciflutrina foi superior aos demais. Todos os ensaios e tratamentos atenderam a NBR 14810-2 (ABNT, 2013), que caracteriza o painel como Tipo P7 (painéis estruturais para uso em condições severas de carga, em ambientes úmidos), exceto o MOE que não atendeu a norma, contudo pode ser classificado como Tipo P5 (painéis estruturais para uso em condições úmidas).

Para as propriedades de resistência a umidade, o tratamento sem preservante e o tratamento DTO apresentaram melhores resultados, tanto no ensaio cíclico quanto no ensaio em água em ebulição. Ambos os ensaios e tratamentos atenderam a NBR 14810-2 (ABNT, 2013).

A resistência ao ataque de cupim de madeira seca, Cryptotermes brevis, na fase normal teve a eficiência comprovada com o tratamento DTO. Na fase bisel o DTO não foi eficiente na inibição do ataque por não ter sido incorporado ao processo produtivo do painel. O DTO não apresentou alta taxa de mortalidade, em ambas as faces, comprovando o seu caráter inibidor e não como inseticida.

O preservante alternativo Distilled Tall Oil apresentou eficiência igual ao preservante Ciflutrina, relacionado a inibição de ataque de cupim de madeira seca Cryptotermes brevis.

\section{REFERÊNCIAS}

ABNT - ASSOCIAÇÃO BRASILEIRA DE NORMAS TÉCNICAS. NBR 14810-2: Painéis de partículas de média densidade. Parte 2 - Requisitos e métodos de ensaio. Rio de Janeiro, 2013. 71 p.

ABNT - ASSOCIAÇÃO BRASILEIRA DE NORMAS TÉCNICAS. NBR 7990: Madeira - Determinação do material solúvel em hidróxido de sódio a 1\%. Rio de Janeiro, 2010a. 4 p.

ABNT - ASSOCIAÇÃO BRASILEIRA DE NORMAS TÉCNICAS. NBR 14853: Madeira - Determinação do material solúvel em etanol:tolueno e em diclorometano e em acetona. Rio de Janeiro, 2010b 3 p.

ABNT - ASSOCIAÇÃO BRASILEIRA DE NORMAS TÉCNICAS. NBR 14660: Madeira - Amostragem e preparação para análise. Rio de Janeiro, 2004. 7 p.

ABNT - ASSOCIAÇÃO BRASILEIRA DE NORMAS TÉCNICAS. NBR 11941: Madeira - Determinação da densidade básica. Rio de Janeiro, 2003. 2 p. 
Antunes et al. - Propriedades tecnológicas de painéis estruruais waferboard

com tratamento distilled tal oil

ABNT - ASSOCIAÇÃO BRASILEIRA DE NORMAS TÉCNICAS. NBR 7988: Madeira - Determinação do teor de extraíveis com água: método de ensaio. Rio de Janeiro, 1984. 3 p.

ASTM - AMERICAN SOCIETY FOR TESTING AND MATERIALS. ASTM D 1037: Standard methods of evaluating properties of wood-base fiber and particles materials. Philadelphia, 2002.

BOSSARDI, K.; BARREIROS, R. M. Extrativos naturais como preservantes para madeiras de rápido crescimento - uma revisão. Revista Ciência da Madeira, v. 2, n. 2, p. 10-129, 2011.

CS - COMMERCIAL STANDARD C.S. 236-66. Mat formed wood particleboard. Geneva, 1968. 18 p.

CSA - CANADIAN STANDARDS ASSOCIATION. CSA 0437.0 - 93: OSB and Waferboard. Ontario, 1993.18 p.

SILVA, F. C.; TREVISAN, H.; SILVA, K. E. D.; LELIS, R. C. C.; CARVALHO, A. G. Influência de diferentes adesivos utilizados na fabricação de painéis aglomerados na ação de Coptotermes gestroi (WASMANN, 1896) (ISOPTERA: RHINOTERMITIDAE). Floresta, v. 40, n. 2, p. 379-384, 2010.

FRANÇA, M. C.; CUNHA, A. B.; TRIANOSKI, R.; SCHIMALSKI, M. B.; RIOS, P. D. Produção de painéis aglomerados homogêneos a partir de fibras oversize residuais de uma indústria de MDF. Scientia Forestalis, v. 44 , p. $665-674,2016$

GALlO, D.; NAKANO, O. SILVEIRA NETO, S.; CARVALHO, R. P. L.; BAPTISTA, G. C.; BERTI FILHO, E.; PARRA, J. R. P.; ZUCHI, R. A.; ALVES, S. B.; VENDRAMIM, J. D.; MARCHINI, L. C.; LOPES, J. R. S.; OMOTO, C. Entomologia Agrícola. São Paulo: FEALQ/USP, 2002. 920 p.

IWAKIRI, S. Painéis de madeira reconstituída. Curitiba: Fupef, 2005. p. 247.

JOSELEAU, J. P.; IMAI, T.; KURODA, L.; RUEL K. Detection in situ and characterization of lignin in the Glayer of tension wood fibres of Populus deltoids. Planta, v. 219, n. 2, p. 338-345, 2004.

KOSKI, A. Applicability of crude tall oil for wood protection faculty of technology. 100 p. 2008 . Tese (Doutorado em Tecnologia) - Finland University of Oulu, Oulu, 2008.

LIMA, C. K. P., MORI, F. A., MENDES, L. M., CARNEIRO, A.C.O. Características anatômicas e química da madeira de clones de Eucalyptus e sua influência na colagem. Cerne, v. 13, n. 2, p. 123-129, 2007.

MALONEY, T. M. Modern particleboard \& dry-process fiberboard manufacturing. San Francisco: Miller Freeman, 1983. 689 p.

MENDES, R. F.; BORTOLETTO J., G.; VIDAL, J. M.; ALMEIDA, N. F.; JANKOWSKY, I. P. Efeito do tratamento preservativo de painéis compensados sobre as suas propriedades físico-mecânicas. Scientia Forestalis, v. 41, n. 100, p. 507-513, 2013.

MENDES, L. M.; IWAKIRI, S.; MATOS, J. L. M.; KEINERT J. S.; SALDANHA, L. K. Pinus spp. na produção de painéis de partículas orientadas (OSB). Ciência Florestal, v. 12, n. 2, p. 135-145, 2002.

PEREIRA, G. F.; RIOS, P. D.; BUSS, R.; VIEIRA, H. C.; GRUBERT, W.; BELINI, U. L. Painéis de madeira aglomerada de Eucalyptus badjensis e Pinus spp. Scientia Forestalis, v. 45, p. 373-382, 2017.

TAPPI - TECHNICAL ASSOCIATION OF THE PULP AND PAPER INDUSTRY. TAPPI T222 om-08: Acid-insoluble lignin in wood and pulp, Test Method. Atlanta, 2002. 5 p.

TAPPI - TECHNICAL ASSOCIATION OF THE PULP AND PAPER INDUSTRY. TAPPI T264 cm-97: preparation of wood for chemical analysis. Atlanta, 1997. 3 p.

TAPPI - TECHNICAL ASSOCIATION OFTHE PULP AND PAPER INDUSTRY. TAPPI 413 om-06: Ash in wood, pulp, paper and paperboard: combustion at $900^{\circ} \mathrm{C}$, Test Method. Atlanta, 1993. 4 p.

USDA - UNITED STATES DEPARTMENT OF AGRICULTURE. Wood handbook: wood as an engineering material. United States Government Printing, 1987. 509 p.

VIDAL, J. M.; EVANGELISTA, W. V.; SILVA, J. C.; JANKOWSKY, I. P. Preservação de madeiras no Brasil: histórico, cenário atual e tendências. Ciência Florestal, v. 25, n. 1, p. 257-271, 2015.

Recebido em: 19/12/2017

Aceito em: 05/12/2018 